\title{
Predictors of sleepiness in obstructive sleep apnoea at baseline and after 6 months of continuous positive airway pressure therapy
}

\author{
Rohit Budhiraja ${ }^{1,2}$, Clete A. Kushida ${ }^{3}$, Deborah A. Nichols ${ }^{3}$, James K. Walsh ${ }^{4}$, \\ Richard D. Simon ${ }^{5}$, Daniel J. Gottlieb ${ }^{1,6}$ and Stuart F. Quan ${ }^{1,7}$
}

Affiliations: ${ }^{1}$ Division of Sleep and Circadian Disorders, Brigham and Women's Hospital, Harvard Medical School, Boston, MA, USA. ${ }^{2}$ Division of Pulmonary and Critical Care Medicine, Dept of Medicine, Brigham and Women's Hospital, Harvard Medical School, Boston, MA, USA. ${ }^{3}$ Stanford University Sleep Clinic and Center for Human Sleep Research, Redwood City, CA, USA. 'Sleep Medicine and Research Center, St Luke's Hospital, Chesterfield, MO, USA. ${ }^{5}$ Providence St Mary Medical Center, Walla Walla, WA, USA. ${ }^{6}$ VA Boston Healthcare System, Boston, MA, USA. ${ }^{7}$ Arizona Respiratory Center, University of Arizona, Tucson, AZ, USA.

Correspondence: Rohit Budhiraja, Division of Sleep and Circadian Disorders and Division of Pulmonary and Critical Care Medicine, Dept of Medicine, Brigham and Women's Hospital, Harvard Medical School, 221 Longwood Avenue, Boston, MA 02115, USA. E-mail: rbudhirajadpartners.org

@ERSpublications

Depression, younger age and higher AHI are associated with sleepiness in people with obstructive sleep apnoea http://ow.ly/GgRL30eG0uC

Cite this article as: Budhiraja R, Kushida CA, Nichols DA, et al. Predictors of sleepiness in obstructive sleep apnoea at baseline and after 6 months of continuous positive airway pressure therapy. Eur Respir J 2017; 50: 1700348 [https://doi.org/10.1183/13993003.00348-2017].

ABSTRACT We evaluated factors associated with subjective and objective sleepiness at baseline and after 6 months of continuous positive airway pressure (CPAP) therapy in patients with obstructive sleep apnoea (OSA).

We analysed data from the Apnoea Positive Pressure Long-term Efficacy Study (APPLES), a prospective 6-month multicentre randomised controlled trial with 1105 subjects with OSA, 558 of who were randomised to active CPAP. Epworth sleepiness scale (ESS) scores and the mean sleep latency (MSL) on the maintenance of wakefulness test at baseline and after 6 months of CPAP therapy were recorded.

Excessive sleepiness (ESS score >10) was present in 543 (49.1\%) participants. Younger age, presence of depression and higher apnoea-hypopnoea index were all associated with higher ESS scores and lower MSL. Randomisation to the CPAP group was associated with lower odds of sleepiness at 6 months. The prevalence of sleepiness was significantly lower in those using CPAP $>4$ h.night ${ }^{-1}$ versus using CPAP $\leqslant 4 \mathrm{~h} \cdot$ night $^{-1}$. Among those with good CPAP adherence, those with ESS $>10$ at baseline had significantly higher odds (OR 8.2, $\mathrm{p}<0.001)$ of persistent subjective sleepiness.

Lower average nightly CPAP use and presence of sleepiness at baseline were independently associated with excessive subjective and objective sleepiness after 6 months of CPAP therapy.

Received: Feb 182017 | Accepted after revision: Aug 192017

Support statement: The Apnea Positive Pressure Long-term Efficacy Study (APPLES) was funded by contract 5UO1-HL-068060 from the National Heart, Lung, and Blood Institute. The APPLES pilot studies were supported by grants from the American Academy of Sleep Medicine and the Sleep Medicine Education and Research Foundation to Stanford University and by the National Institute of Neurological Disorders and Stroke (N44-NS-002394) to SAM Technology. Funding information for this article has been deposited with the Crossref Funder Registry.

Conflict of interest: Disclosures can be found alongside this article at erj.ersjournals.com

Copyright OERS 2017 


\section{Introduction}

Excessive daytime sleepiness (EDS) is frequently reported in patients with obstructive sleep apnoea (OSA). Conversely, OSA has been suggested to be the most common medical disorder that causes EDS [1]. A variety of factors may be associated with EDS in OSA patients. Several studies have assessed the association between the apnoea-hypopnoea index (AHI) and sleepiness, but while some studies found an association between AHI and EDS [2-5], others did not [6-8]. In addition, obesity, oxygen desaturation index and depressive symptoms have been associated with EDS in OSA patients [9-11]. However, many of these studies had small sample sizes or did not have objective means of assessment such as the mean sleep latency (MSL) test or maintenance of wakefulness test (MWT). Furthermore, while several medical and psychiatric disorders have been associated with poor sleep quality and insomnia [12-14], there are few investigations assessing the impact of comorbid disorders on sleepiness in OSA. Finally, there are few large studies assessing the factors associated with residual sleepiness in patients on continuous positive airway pressure (CPAP) therapy.

The Apnoea Positive Pressure Long-term Efficacy Study (APPLES) was a prospective multicentre randomised controlled trial designed to assess the impact of CPAP on several domains of neurocognitive outcomes in adults with OSA. Participants with OSA were randomised to either active CPAP or sham CPAP for 6 months. The subjects completed a number of questionnaires including the Epworth sleepiness scale (ESS) and underwent several tests including the MWT at baseline and 6 months after initiation of CPAP therapy.

In this report, we evaluated the prevalence of subjective and objective sleepiness as well as demographic and polysomnographic variables and medical and psychiatric conditions associated with sleepiness in participants with OSA. We also assessed the factors associated with sleepiness after 6 months of CPAP therapy. Specifically, we aimed to evaluate the impact of different levels of CPAP adherence on prevalence of sleepiness.

\section{Methods}

\section{Study design}

The APPLES protocol has been described in detail elsewhere [15]. In brief, participants were recruited from sleep clinics and through public advertisements at five sites in the USA: Stanford University (Stanford, CA), University of Arizona (Tucson, AZ), Providence St Mary Medical Center (Walla Walla, WA), St Luke's Hospital (Chesterfield, MO) and Brigham and Women's Hospital (Boston, MA). Those who did not have exclusion criteria during the initial interview and consented to participate in the study underwent diagnostic polysomnography (PSG). Those who had an AHI $\geqslant 10$ events $\cdot \mathrm{h}^{-1}$ without severe oxygen desaturation (i.e. oxygen saturation $<75 \%$ for $>10 \%$ of the diagnostic sleep study) were randomised to either active or sham CPAP (REMStar Pro; Phillips Respironics, Murrysville, PA, USA) for 6 months. The participants randomised to active CPAP then underwent a titration in the sleep laboratory to determine the optimal therapeutic pressure. A sham titration was performed for those randomised to sham CPAP. Although participants' adherence was monitored continuously during the study, for this report we calculated adherence at the 6-month time point after initiation of CPAP.

\section{Participants}

Out of the 1516 participants screened, 1105 were ultimately randomised (268 participants were removed from study pre-randomisation due to exclusion criteria (e.g. taking exclusionary medication) and 143 participants withdrew from the study pre-randomisation for any reason (e.g. too busy)) [15], with 558 assigned to active CPAP and 547 assigned to sham CPAP. Heated humidification was used in all participants. Staff (e.g. respiratory therapists, sleep technologists or physicians) worked in a systematic manner to troubleshoot nasal congestion and other problems that may have been reported by a participant. Subjects using hypnotics, anxiolytics, sedating antidepressants, anticonvulsants, sedating antihistamines, stimulants or other medications likely to affect neurocognitive function and/or alertness were excluded from the study. Some subjects were on antidepressants and some on opioids, including those in antitussive medications.

\section{Pre-existing medical conditions}

At the baseline visit, all participants underwent a clinical evaluation including a physical examination to obtain a medical history and information pertaining to symptoms of sleep disorders, and presence of other medical and psychiatric conditions such as cardiovascular disease, gastro-oesophageal reflux disease (GORD), chronic pain, nasal congestion and depression. A condition was considered present when the participant answered that they currently had it. Furthermore, the Hamilton rating scale for depression (HAMD) [16] was administered at baseline and at the 6-month post-CPAP follow-up visit. We considered a score $\geqslant 8$ as indicative of depression being present. 
Epworth sleepiness scale

The ESS is one of the most widely used assessments of subjective daytime sleepiness $[17,18]$. It is a self-administered questionnaire where individuals rate their usual chances of dozing off or falling asleep in eight common situations or activities on a 4-point scale (0-3). Hence, the minimum possible score on the scale is 0 (not sleepy at all) and the maximum is 24 (extremely sleepy). The ESS was administered at baseline and at 6 months after starting active CPAP or sham CPAP. Sleepiness was considered clinically significant if the ESS total score was $>10$.

\section{Maintenance of wakefulness test}

The MWT test is used to assess objective daytime sleepiness [18, 19]. With the participant sitting in bed with eyes open, it measures sleepiness when the intention is to stay awake. Hence, it provides an objective estimate of the propensity to fall asleep when people would want to stay awake during their normal day. The MWT was performed at baseline and at the 2- and 6-month follow-up visits. For this study, we used the tests performed at the baseline and 6-month follow-up visits. Briefly, after overnight PSG (diagnostic at baseline and with patient's own CPAP at 6 months), four 20-min trials were conducted at 10:00 h, 12:00 h, 14:00 $\mathrm{h}$ and 16:00 h. A trial was ended after $20 \mathrm{~min}$ or after three consecutive 30-s epochs of any stage of sleep, whichever occurred first. Once a trial was terminated, the room lights were turned on and the subject was asked to open his or her eyes, stay seated in bed and remain awake until the 20 min of the trial had elapsed. The MSL of all four trials was used for these analyses. Inter-rater reliability assessments were conducted for PSGs and MWTs blindly scored by data coordinating centre PSG technologists, and the data coordinating centre was responsible for further quality assurance/quality control procedures for these data [15].

\section{CPAP adherence}

Adherence to active CPAP or sham CPAP was measured objectively using Encore Pro SmartCards (Phillips Respironics) that were exchanged at the clinic twice monthly. For this report, at the 6-month period after start of CPAP, the mean hours of daily use were analysed for the preceding 2-month period to assess adherence during that time interval. The SmartCard is a memory card that records data regarding the duration of each use of CPAP therapy and provides reliable objective evidence of CPAP use.

\section{Statistical analyses}

To assess the factors associated with sleepiness at baseline, we included all 1105 participants. For the 6-month analyses, we focused only on those randomised to active CPAP therapy with relevant data available. For assessment of residual sleepiness in those with good adherence, analyses were limited to those participants who were using CPAP $>4 \mathrm{~h} \cdot$ night $^{-1}$. For continuous variables, unadjusted comparisons between groups were made using unpaired t-tests. Data were expressed as mean $\pm \mathrm{SD}$. Differences in proportions were assessed using the Chi-squared test. Logistic regression was used to assess odds ratios $(95 \% \mathrm{CI})$ for sleepiness (ESS $>10)$ at baseline and at 6 months after CPAP initiation, with predictors of interest including demographic and polysomnographic characteristics and comorbid conditions. A multiple regression model was used to examine the association between the ESS scores and MSL values as dependent variables, with demographic and polysomnographic characteristics and comorbid conditions at baseline and at 6 months. The statistical significance level was set at $\mathrm{p}<0.05$ (two-tailed) for all tests. Statistical analyses were conducted using SPSS version 20.0 for Windows (SSPS, Chicago, IL, USA).

\section{Results}

\section{Sleepiness at baseline}

1105 participants were assessed, comprising 382 females and 723 males, of whom 611 (55\%) had severe OSA defined as AHI $>30$ events $\cdot \mathrm{h}^{-1}$. The baseline demographics for all participants and subsequently randomised participants stratified by sex are provided in an earlier publication [20]. Briefly, the mean age of the participants was $51.5 \pm 12.2$ years, mean body mass index (BMI) was $32.2 \pm 7.1 \mathrm{~kg} \cdot \mathrm{m}^{-2}$, mean AHI was $38.2 \pm 26.7$ events $\cdot \mathrm{h}^{-1}$ and the mean oxygen desaturation index was $25.5 \pm 25.1$ events $\cdot \mathrm{h}^{-1}$. The mean baseline ESS score of the participants was 10.4 \pm 4.4 (minimum 0, maximum 22). In bivariate analyses, females were significantly sleepier (ESS score $10.9 \pm 4.5$ versus $10.2 \pm 4.3, \mathrm{p}=0.006$ ) than males. There was a trend for those with severe sleep apnoea to be sleepier than those without severe sleep apnoea (ESS score $10.7 \pm 4.5$ versus $10.2 \pm 4.3, \mathrm{p}=0.07$ ). The ESS scores correlated directly with BMI, HAMD score and several polysomnographic variables, including AHI, and inversely with age (table 1). Of the 1105 participants, 543 $(49.1 \%)$ had excessive sleepiness (ESS score $>10$ ). As shown in table 2 , the ESS total scores as well as proportion of participants who were sleepy (ESS score $>10$ ) were higher in those with depression and chronic pain. Except for a trend towards greater sleepiness in those with GORD, no differences were observed in a variety of other medical and psychiatric conditions. Of all subjects, 196 were on 
TABLE 1 Bivariate correlation between clinical and polysomnographic variables and the Epworth sleepiness scale total score at baseline

\begin{tabular}{lcc} 
& Pearson correlation & p-value \\
\hline Age & -0.07 & 0.02 \\
BMI & 0.10 & 0.001 \\
AHI & 0.08 & 0.01 \\
Desaturation index & 0.08 & 0.01 \\
Sleep efficiency & 0.09 & 0.02 \\
HAMD total score & 0.13 & $<0.001$ \\
\hline
\end{tabular}

BMI: body mass index; AHI: apnoea-hypopnoea index; HAMD: Hamilton rating scale for depression.

antidepressants and 73 were on opiates. The use of antidepressants $(p=0.31)$ or opiates $(p=0.15)$ was not significantly associated with greater sleepiness.

A linear regression model revealed younger age, higher AHI and elevated HAMD scores to be independently associated with higher ESS total scores (table 3). Logistic regression showed higher odds of sleepiness in those with depression (HAMD scores $\geqslant 8$, OR $1.4 ; \mathrm{p}=0.03$ ) and lower odds with increasing age (table 4).

The MWT MSL at baseline (MSL-B; $\mathrm{n}=1086$ ) was $17.0 \mathrm{~min}$ (range 1.9-20 min). An MSL-B of 20 min was seen in $521(48 \%)$ participants and an MSL-B <20 min was seen in 565 (52\%) participants. For these analyses, a MSL $<20$ min was considered abnormal. Notably, we repeated analyses using MSL $\leqslant 17 \mathrm{~min}$ as abnormal, which was seen in $37.5 \%(\mathrm{n}=407)$ of all participants at baseline. The results obtained using this cut-off to define sleepiness were generally similar to those obtained using MSL $<20 \mathrm{~min}$ as abnormal.

As expected, MSL-B was significantly lower in those with ESS score $>10$ than those with ESS scores $\leqslant 10$ $(16.3 \pm 4.3 \mathrm{~min}$ versus $17.7 \pm 3.5 \mathrm{~min}, \mathrm{p}<0.001)$. Of those with ESS scores $>10,61.2 \%$ had a MSL-B $<20 \mathrm{~min}$ compared to only $43.2 \%$ in those with an ESS score $\leqslant 10(p<0.001)$. Logistic regression showed that younger age, presence of depression and AHI $>30$ events $^{-1} h^{-1}$ were associated with higher odds of sleepiness (MSL-B <20 min) (table 5).

\section{Sleepiness at 6 months after CPAP initiation}

The mean \pm SD age of the participants randomised to CPAP was $52.2 \pm 12.1$ years, BMI was $32.3 \pm 7.4 \mathrm{~kg} \cdot \mathrm{m}^{-2}$, AHI was $39.5 \pm 24.9$ events $\cdot \mathrm{h}^{-1}$ and oxygen desaturation index was $26.3 \pm 24.1$ events $\cdot \mathrm{h}^{-1}$. At 6 months, the AHI in this group was $6.1 \pm 8.3$ events $\cdot h^{-1}$ and the mean oxygen desaturation index was $4.8 \pm 7.3$ events $\cdot h^{-1}$. In contrast, the mean AHI in the sham group at 6 months was $29.8 \pm 25.1$ events $\cdot h^{-1}$ and the mean oxygen desaturation index was $24.8 \pm 24.9$ events $\mathrm{h}^{-1}$. ESS score was significantly lower in the CPAP group compared to the sham group at 2 months (7.9 versus $8.8, \mathrm{p}=0.003), 4$ months $(7.0$ versus $8.2, \mathrm{p}<0.001)$ and 6 months $(7.3$ versus $8.4, \mathrm{p}=0.003)$ after initiation of therapy, despite similar ESS scores at baseline $(10.5$ versus $10.5, \mathrm{p}=0.99)$.

At 6 months, 88 (22.3\%) of the 394 participants initiated on CPAP had excessive sleepiness defined as ESS $>10$. The mean nightly adherence at 6 months in those randomised to active CPAP was $4.70 \pm 2.04 \mathrm{~h}$ and $67.3 \%$ of the participants were using CPAP $>4 \mathrm{~h} \cdot$ night $^{-1}$. Further details of adherence in the study participants are provided in an earlier publication [21]. Generally, the prevalence of sleepiness declined progressively with increasing CPAP use (figure 1). The prevalence of sleepiness was higher in those using

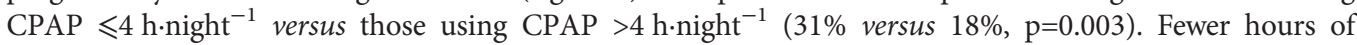
CPAP use and higher ESS total scores at baseline were independently associated with higher 6-month ESS total scores in a linear regression model (table 6). A logistic regression model (table 7) showed lower odds of sleepiness in those using CPAP $>4 \mathrm{~h} \cdot$ night $^{-1}$ (OR 0.42, p=0.001), and higher odds in those who were sleepy at baseline (OR 5.1, $\mathrm{p}<0.001)$. Females had lower odds of sleepiness than males; the presence of chronic pain was associated with higher odds of sleepiness; and the presence of depression was associated with a trend towards higher odds of sleepiness (table 7). The use of antidepressants $(\mathrm{n}=100, \mathrm{p}=0.4)$ or opiates $(\mathrm{n}=38, \mathrm{p}=0.08$ ) was not significantly associated with ESS scores $>10$.

MWT and CPAP adherence data were available for 380 participants randomised to CPAP, 6 months after initiation of therapy. MSL on MWT at 6 months (MSL-6m) was $18 \mathrm{~min}$. MSL- $6 \mathrm{~m}$ was significantly greater

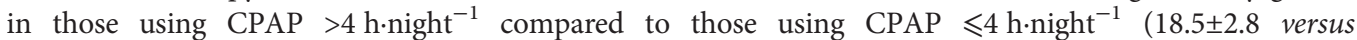
$17.5 \pm 3.8 \mathrm{~min} ; \mathrm{p}=0.01)$. MSL- $6 \mathrm{~m}$ was $<20 \mathrm{~min}$ in $147(38.7 \%)$ participants and $\leqslant 17 \mathrm{~min}$ in $90(23.7 \%)$ participants. Logistic regression revealed significantly lower odds of excessive sleepiness, defined as 
TABLE 2 The mean Epworth sleepiness scale (ESS) total score and proportion of sleepy participants in various medical and psychiatric disorders

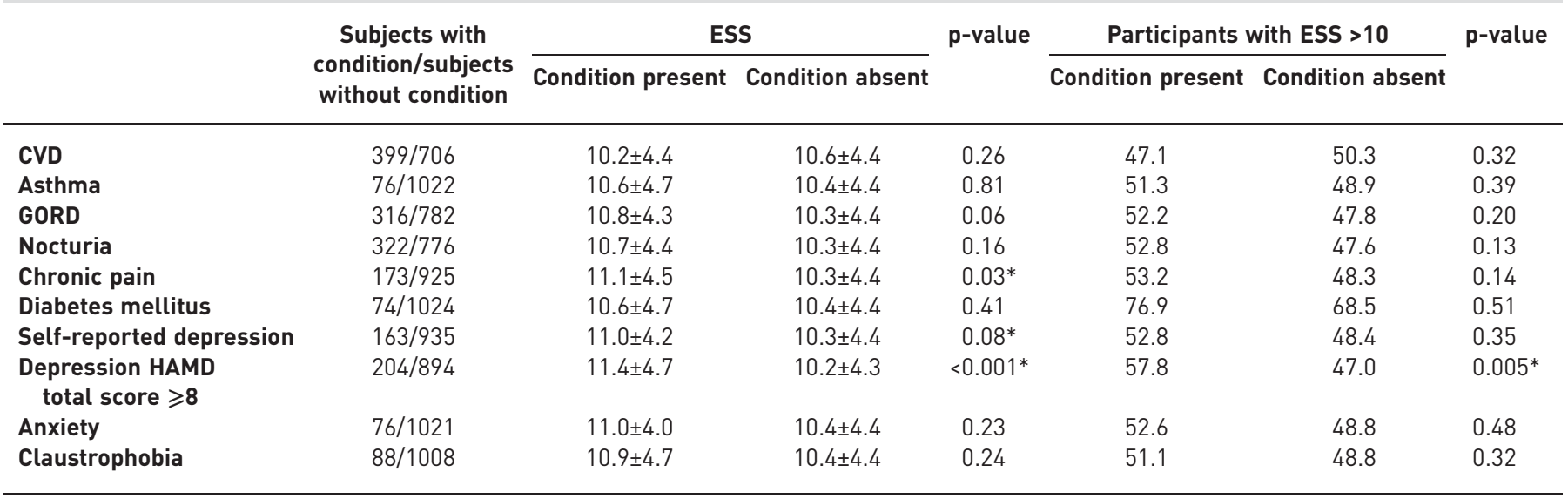

Data are presented as $\mathrm{n} / \mathrm{n}$, mean $\pm \mathrm{SD}$ or \%, unless otherwise stated. CVD: cardiovascular disease; GORD: gastro-oesophageal reflux disease; HAMD: Hamilton rating scale for depression. *: $p<0.05$.

MSL-6m $<20 \mathrm{~min}$, in those using CPAP $>4 \mathrm{~h} \cdot$ night $^{-1}$ (OR 0.55, $\left.\mathrm{p}=0.008\right)$. Those with MSL-B $<20 \mathrm{~min}$ had significantly higher odds of MSL- $6 \mathrm{~m}<20 \mathrm{~min}(\mathrm{OR}=6.7, \mathrm{p}<0.001)$ (table 8). Using a cut-off of $17 \mathrm{~min}$ for MSL-6m to define sleepiness yielded similar results.

Sleepiness at 6 months in participants with good adherence to CPAP

The prevalence of ESS score $>10$ was $18.1 \%$ (48 out of 265 subjects) among those using CPAP $>4$ h. night $^{-1}$.

The presence of excessive sleepiness at 6 months in these patients with good CPAP adherence was higher in those with baseline excessive sleepiness, whether assessed by ESS score $>10$ (OR 8.2, $\mathrm{p}<0.001$ ) or MSL-B $<20$ min (OR 9.6, p<0.001). No other variables assessed, including age, BMI, AHI on the sleep study performed at 6 months or presence of depression, chronic pain or GORD were significantly associated with odds of sleepiness at 6 months in this group of patients.

\section{Discussion}

The current study found that excessive sleepiness, as measured by ESS total score $>10$ or a MSL $<20$ min on MWT, was present in half the patients with sleep apnoea, and was associated with presence of depression, more severe sleep apnoea and younger age. The prevalence of excessive sleepiness 6 months after initiating CPAP was greatly reduced to $22.3 \%$, and was inversely associated with mean hours of CPAP use. Presence of excessive sleepiness at baseline was a strong determinant of excessive sleepiness after 6 months of therapy, even in those who had good adherence to CPAP.

We found that younger age, higher AHI and presence of depression are associated with increased sleepiness in patients with sleep apnoea. Past literature assessing an association between AHI and EDS has

TABLE 3 Linear regression analysis with Epworth sleepiness scale total score as the
dependent variable
\begin{tabular}{lccccc} 
& $\boldsymbol{B}$ & SE & Standardised coefficient $\boldsymbol{\beta}$ & $\mathbf{t}$ & p-value \\
\hline Age & -0.025 & 0.011 & -0.068 & -2.211 & $0.027^{*}$ \\
Female & 0.530 & 0.292 & 0.057 & 1.812 & 0.070 \\
BMI & 0.021 & 0.021 & 0.033 & 0.984 & 0.325 \\
AHI & 0.012 & 0.006 & 0.066 & 2.009 & $0.045^{*}$ \\
HAMD total score $\geqslant 8$ & 0.118 & 0.033 & 0.109 & 3.556 & $<0.001^{*}$ \\
Chronic pain & 0.609 & 0.373 & 0.050 & 1.631 & 0.103 \\
GORD & 0.364 & 0.297 & 0.037 & 1.227 & 0.220
\end{tabular}

BMI: body mass index; AHI: apnoea-hypopnoea index; HAMD: Hamilton rating scale for depression; GORD: gastro-oesophageal reflux disease. ${ }^{*}:$ p $<0.05$. 


\begin{tabular}{|c|c|c|c|c|c|}
\hline & ESS score > 10 & ESS score $<10$ & Wald & Odds ratio & p-value \\
\hline Subjects & 543 & 562 & & & \\
\hline Age years & $50.4 \pm 11.9$ & $52.7 \pm 12.4$ & 9.318 & 0.984 & $0.002 *$ \\
\hline Female & 206 (37.9) & 176 (31.3) & 3.493 & 1.290 & 0.062 \\
\hline BMI $\mathrm{kg} \cdot \mathrm{m}^{-2}$ & $32.7 \pm 6.9$ & $31.7 \pm 7.3$ & 0.108 & 1.003 & 0.742 \\
\hline Baseline AHI $>30$ events $\cdot \mathrm{h}^{-1}$ & 312 (57.5) & $299(53.2)$ & 2.974 & 1.005 & 0.085 \\
\hline HAMD total score $\geqslant 8$ & $118(21.9)$ & $86(15.4)$ & 4.732 & 1.423 & $0.030 *$ \\
\hline Chronic pain & $92(17.1)$ & $81(14.5)$ & 0.624 & 1.148 & 0.429 \\
\hline GORD & $165(30.6)$ & $151(27.0)$ & 1.010 & 1.149 & 0.315 \\
\hline
\end{tabular}

Data are presented as $n$, mean \pm SD or $n(\%)$, unless otherwise stated. BMI: body mass index; AHI: apnoeahypopnoea index; HAMD: Hamilton rating scale for depression; GORD: gastro-oesophageal reflux disease. $*$ : $p<0.05$.

shown conflicting results, with some studies [2-5], but not others [6-8] showing an association. The studies that have not shown this association probably reflect a referral bias, since sleepy patients are more likely to be referred for sleep evaluation, thereby obscuring the relationship of AHI and sleepiness seen in population-based studies.

The association between depression and increased sleepiness confirms the findings of prior reports [10, 22, 23]. Depression is a common finding in patients with OSA. We found an $18.6 \%$ prevalence of depression, defined as HAMD score $\geqslant 8$. This is similar to a $20.8 \%$ prevalence of depression found in patients with untreated OSA in a recent study [22]. Similar to that study, we found an association between depression and ESS. This presents a strong argument for making appropriate investigations into the presence of depression in those with sleep disordered breathing, especially in the presence of sleepiness.

ESS scores and prevalence of sleepiness decreased with increasing age, as has been reported in some prior studies $[23,24]$. This may be related to less potent sleep homeostasis mechanisms with increasing age, including morphological and neurological changes in suprachiasmatic nucleus and a reduction in melatonin concentration with ageing [25]. These findings suggest that complaints of sleepiness in the elderly would represent a less common response, and should not be considered a normal part of the physiological ageing process.

Lower average nightly CPAP use and presence of sleepiness at baseline were the primary determinants of residual sleepiness identified in this study. The effect of good adherence on reducing sleepiness confirms the benefit of this therapy, as demonstrated in past studies [26-28]. However, we found that after 6 months of CPAP therapy, $22.3 \%$ of the participants on CPAP $\left(18.1 \%\right.$ of those using CPAP $>4 \mathrm{~h} \cdot$ night $\left.^{-1}\right)$ still had sleepiness. This prevalence is close to that noted in recent studies $[27,28]$, and is similar to the prevalence of excessive sleepiness in the general population among those without sleep apnoea [4]. Thus, while increasing CPAP adherence beyond $4 \mathrm{~h} \cdot$ night $^{-1}$ might yield some further reduction in daytime sleepiness, much of the residual sleepiness in these patients may be caused by factors other than OSA,

TABLE 5 Odds of objective sleepiness (maintenance of wakefulness test (MWT) $<20 \mathrm{~min}$ ) at baseline

\begin{tabular}{lcccc} 
& MWT<20 $\mathbf{~ m i n}$ & MWT=20 $\mathbf{~ m i n}$ & Odds ratio & p-value \\
\hline Subjects & 521 & 565 & & \\
Age years & $49.6 \pm 12.6$ & $53.8 \pm 11.3$ & 0.972 & $<0.001^{*}$ \\
Female & $196(34.7)$ & $178(34.2)$ & 0.977 & 0.868 \\
BMI $\mathbf{~ k g} \cdot \mathbf{m}^{-\mathbf{2}}$ & $33.1 \pm 7.4$ & $31.2 \pm 6.6$ & 1.018 & 0.064 \\
Baseline AHI $>\mathbf{3 0}$ events $\cdot \mathbf{h}^{-1}$ & $354(62.7)$ & $249(47.8)$ & 1.858 & $<0.001^{*}$ \\
HAMD total score $\geqslant \mathbf{8}$ & $123(21.9)$ & $79(15.2)$ & 1.500 & $0.016^{*}$ \\
Chronic pain & $91(16.1)$ & $81(15.5)$ & 1.081 & 0.664 \\
GORD & $161(28.5)$ & $149(28.6)$ & 0.921 & 0.563 \\
\hline
\end{tabular}

Data are presented as $n$, mean \pm SD or $n(\%)$, unless otherwise stated. BMI: body mass index; AHI: apnoeahypopnoea index; HAMD: Hamilton rating scale for depression; GORD: gastro-oesophageal reflux disease. $*$ : $p<0.05$. 


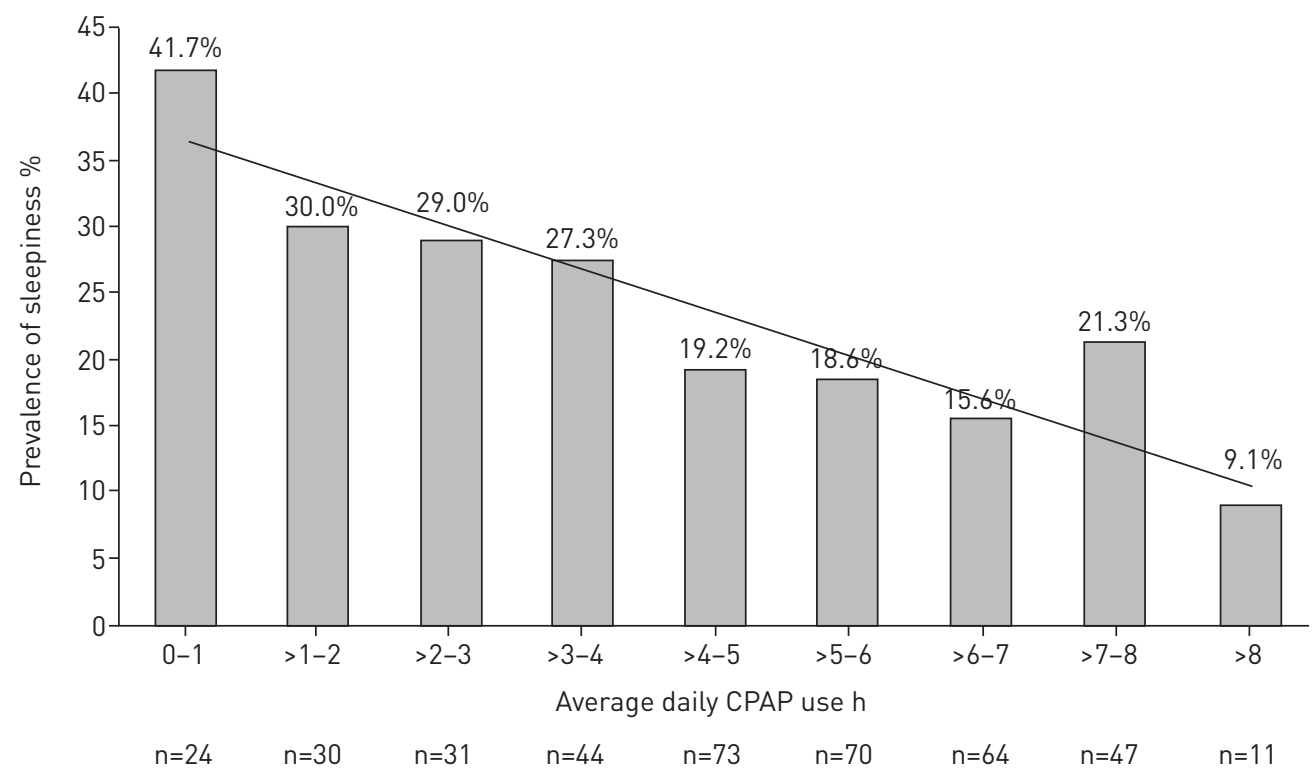

FIGURE 1 The prevalence of sleepiness (Epworth sleepiness scale score $>10$ ) in all participants with different mean daily continuous positive airway pressure (CPAP) use.

indicating the importance of evaluating patients with residual sleepiness on CPAP for alternative causes of sleepiness. Oxidative injury to sleep-wake brain regions from long-term intermittent hypoxia has been suggested as a reason for residual sleepiness following treatment of OSA [29]. However, reasons for sleepiness, such as chronic pain or other conditions contributing to repeated wakefulness at night, sedative medications, depression, poor sleep hygiene or insufficient habitual sleep duration should be sought and modified before attributing residual sleepiness to a failure of CPAP therapy. In view of the finding that those who were sleepy at baseline had significantly higher odds (OR 8.2, $\mathrm{p}<0.001$ ) of sleepiness despite good CPAP adherence, it is imperative that these patients be closely followed to ensure improvement in sleepiness.

The study has several limitations. First, we used the ESS for assessment of sleepiness in patients with OSA. The ESS, while a commonly used measure of sleepiness, has only a limited correlation with objective sleepiness measurements [30]. However, we also used the MWT, an objective measurement of ability to resist sleep onset, and the results were similar to those obtained using subjective measurements from the ESS. Second, while we used MWT sleep latency to objectively assess ability to maintain wakefulness, the optimal MWT cut-off values in those with OSA have not been defined. To overcome this limitation, we

TABLE 6 Linear regression analysis with Epworth sleepiness scale (ESS) total score at 6 months as the dependent variable

\begin{tabular}{lrrrrc} 
& $\boldsymbol{\beta}$ & SE & Standardised coefficient $\boldsymbol{\beta}$ & $\mathbf{t}$ & $\mathbf{p}$-value \\
\hline Age & 0.020 & 0.015 & 0.058 & 1.328 & 0.185 \\
Female & -0.548 & 0.374 & -0.062 & -1.466 & 0.143 \\
BMI & -0.031 & 0.025 & -0.055 & -1.210 & 0.227 \\
HAMD total score & 0.023 & 0.040 & 0.023 & 0.566 & 0.571 \\
Chronic pain & 0.766 & 0.462 & 0.069 & 1.660 & 0.098 \\
GORD & 0.045 & 0.380 & 0.005 & 0.119 & 0.906 \\
Baseline ESS & 0.495 & 0.039 & 0.528 & 12.679 & $<0.001^{*}$ \\
AHI at baseline & -0.007 & 0.008 & -0.038 & -0.841 & 0.401 \\
AHI at 6 months & -0.025 & 0.022 & -0.049 & -1.144 & 0.253 \\
Mean hours of CPAP adherence & -0.240 & 0.067 & -0.151 & -3.579 & $<0.001^{*}$
\end{tabular}

BMI: body mass index; HAMD: Hamilton rating scale for depression; GORD: gastro-oesophageal reflux disease; AHI: apnoea-hypopnoea index; CPAP: continuous positive airway pressure. *: $p<0.05$. 


\begin{tabular}{|c|c|c|c|c|}
\hline & ESS score $>10$ & ESS score $<10$ & Odds ratio & p-value \\
\hline Subjects & 88 & 306 & & \\
\hline Age years & $51.2 \pm 12.5$ & $53.0 \pm 12.0$ & 1.001 & 0.920 \\
\hline Female & $29(33.0)$ & $106(34.6)$ & 0.582 & $0.049 *$ \\
\hline $\mathrm{BMI} \mathrm{kg} \cdot \mathrm{m}^{-2}$ & $31.6 \pm 6.8$ & $32.2 \pm 7.3$ & 0.982 & 0.356 \\
\hline HAMD total score $\geqslant 8$ & $19(21.6)$ & $51(16.7)$ & 1.766 & 0.059 \\
\hline Chronic pain & $23(26.1)$ & 45 (14.7) & 2.289 & $0.008 *$ \\
\hline GORD & $29(33.0)$ & $86(28.1)$ & 0.979 & 0.939 \\
\hline Baseline ESS score $>10$ & $65(73.9)$ & $127(41.5)$ & 5.143 & $<0.001 *$ \\
\hline Baseline $A H I>30$ events $\cdot h^{-1}$ & $48(54.5)$ & $171(55.9)$ & 1.106 & 0.715 \\
\hline $\mathrm{AHI}>5$ events $\cdot \mathrm{h}^{-1}$ at 6 months & $27(30.7)$ & $111(36.3)$ & 0.754 & 0.291 \\
\hline CPAP use $>4 \mathrm{~h} \cdot$ night $^{-1}$ & $48(54.5)$ & $217(70.9)$ & 0.425 & $0.001 *$ \\
\hline
\end{tabular}

Data are presented as $n$, mean \pm SD or $n(\%)$, unless otherwise stated. BMI: body mass index; HAMD: Hamilton rating scale for depression; GORD: gastro-oesophageal reflux disease; AHI: apnoea-hypopnoea index; CPAP: continuous positive airway pressure. ${ }^{*}: p<0.05$.

used different cut-off values to decide whether the patients had decreased wakefulness. Notably, we obtained similar predictors of sleepiness while using these different cut-offs, and the results were consistent with those obtained using ESS. Third, the information about medical and psychiatric conditions was self-reported in most cases and obtained only at baseline. Hence, whether the conditions were present at the 6-month evaluation period, and thus may have influenced sleepiness, is not clear. Furthermore, apart from depression, for which we had HAMD scale values, the severity of other conditions was not assessed. Fourth, residual confounding by several factors including habitual sleep duration, disorders not documented in the study, medications and genetic and socioeconomic factors cannot be excluded [31]. Finally, the standard MWT administration consists of 40-min nap opportunities, while the naps in this trial lasted only $20 \mathrm{~min}$. However, the resultant ceiling effect is expected to reduce power to detect effects of treatment and makes the differences with therapy even more compelling.

In conclusion, this study shows that depression, younger age and higher AHI are associated with sleepiness in people with OSA. CPAP use is associated with reduced odds of sleepiness at 6 months. Lower average nightly CPAP use and presence of sleepiness at baseline are the primary determinants of persistent sleepiness in those using CPAP therapy, even among CPAP-adherent patients. Specific strategies targeting these patients, including stressing the role of CPAP in decreasing sleepiness, frequent follow-up clinic visits to evaluate residual sleepiness and promote adherence [21, 32], as well as thorough evaluation for other aetiologies of sleepiness should be strongly considered. An appreciable number of OSA patients will

TABLE 8 Odds of objective sleepiness (mean sleep latency at 6 months (MSL- $6 \mathrm{~m}$ ) $<20$ min)

\begin{tabular}{|c|c|c|c|c|}
\hline & \multicolumn{2}{|c|}{ MSL-6m } & \multirow[t]{2}{*}{ Odds ratio } & \multirow[t]{2}{*}{ p-value } \\
\hline & $<20 \mathrm{~min}$ & $=20 \mathrm{~min}$ & & \\
\hline Subjects & 147 & 233 & & \\
\hline Age years & $53.8 \pm 12.4$ & $53.8 \pm 11.4$ & 0.994 & 0.5210 \\
\hline Female & 46 (31.3) & $84(36.1)$ & 0.678 & 0.109 \\
\hline BMI $\mathrm{kg} \cdot \mathrm{m}^{-2}$ & $32.2 \pm 6.7$ & $31.9 \pm 7.2$ & 1.004 & 0.794 \\
\hline HAMD total score $\geqslant 8$ & $30(20.4)$ & $39(16.7)$ & 1.168 & 0.587 \\
\hline Chronic pain & 24 (16.3) & 43 (18.5) & 0.827 & 0.524 \\
\hline GORD & 49 (33.3) & 65 (27.9) & 1.249 & 0.364 \\
\hline Baseline MWT $<20 \mathrm{~min}$ & $156(67.0)$ & $33(22.4)$ & 6.711 & $<0.001^{*}$ \\
\hline Baseline $\mathrm{AHI}>30$ events $\cdot \mathrm{h}^{-1}$ & $80(54.4)$ & $128(54.9)$ & 0.811 & 0.398 \\
\hline $\mathrm{AHI}>5$ events $\cdot \mathrm{h}^{-1}$ at 6 months & $51(34.7)$ & $84(36.1)$ & 1.020 & 0.933 \\
\hline CPAP use $>4 \mathrm{~h} \cdot$ night $^{-1}$ & $85(57.8)$ & 169 (72.5) & 0.547 & $0.008 *$ \\
\hline
\end{tabular}

Data are presented as $\mathrm{n}$, mean $\pm \mathrm{SD}$ or $\mathrm{n}(\%)$, unless otherwise stated. $\mathrm{BMI}$ : body mass index; HAMD: Hamilton rating scale for depression; GORD: gastro-oesophageal reflux disease; MWT: maintenance of wakefulness test; AHI: apnoea-hypopnoea index; CPAP: continuous positive airway pressure. *: $p<0.05$. 
continue to have sleepiness despite using CPAP. Other causes of sleepiness and other measures including addition of wake-promoting medications should be considered in these patients.

\section{Acknowledgements}

The Apnea Positive Pressure Long-term Efficacy Study (APPLES) investigators gratefully recognise the vital input and support of Sylvan Green, who died before the results of this trial were analysed, but was instrumental in its design and conduct.

Administrative core: Clete A. Kushida, Deborah A. Nichols, Eileen B. Leary, Pamela R. Hyde, Tyson H. Holmes, Daniel A. Bloch and William C. Dement. Data Coordinating Center: Daniel A. Bloch, Tyson H. Holmes, Deborah A. Nichols, Rik Jadrnicek (Microflow), Ric Miller (Microflow), Usman Aijaz, Aamir Farooq, Darryl Thomander, Chia-Yu Cardell, Emily Kees, Michael E. Sorel, Oscar Carrillo, Tami Crabtree, Booil Jo, Ray Balise and Tracy Kuo. Clinical coordinating centre: Clete A. Kushida, William C. Dement, Pamela R. Hyde, Rhonda M. Wong, Pete Silva, Max Hirshkowitz, Alan Gevins, Gary Kay, Linda K. McEvoy, Cynthia S. Chan and Sylvan Green.

Clinical centres: Stanford University (Stanford, CA, USA): Christian Guilleminault, Eileen B. Leary, David Claman, Stephen Brooks, Julianne Blythe, Jennifer Blair, Pam Simi, Ronelle Broussard, Emily Greenberg, Bethany Franklin, Amirah Khouzam, Sanjana Behari Black, Viola Arias, Romelyn Delos Santos and Tara Tanaka; University of Arizona (Tucson, AZ, USA): Stuart F. Quan, James L. Goodwin, Wei Shen, Phillip Eichling, Rohit Budhiraja, Charles Wynstra, Cathy Ward, Colleen Dunn, Terry Smith, Dane Holderman, Michael Robinson, Osmara Molina, Aaron Ostrovsky, Jesus Wences, Sean Priefert, Julia Rogers, Megan Ruiter and Leslie Crosby; St Mary Medical Center (Walla Walla, WA, USA): Richard D. Simon Jr, Kevin Hurlburt, Michael Bernstein, Timothy Davidson, Jeannine Orock-Takele, Shelly Rubin, Phillip Smith, Erica Roth, Julie Flaa, Jennifer Blair, Jennifer Schwartz, Anna Simon and Amber Randall; St Luke's Hospital (Chesterfield, MO, USA): James K. Walsh, Paula K. Schweitzer, Anup Katyal, Rhody Eisenstein, Stephen Feren, Nancy Cline, Dena Robertson, Sheri Compton, Susan Greene, Kara Griffin and Janine Hall; Brigham and Women's Hospital (Boston, MA, USA): Daniel J. Gottlieb, David P. White, Denise Clarke, Kevin Moore, Grace Brown, Paige Hardy, Kerry Eudy, Lawrence Epstein and Sanjay Patel; We thank Sleep HealthCenters for the use of their clinical facilities to conduct this research.

Consultant Teams: methodology team: Daniel A. Bloch, Sylvan Green, Tyson H. Holmes, Maurice M. Ohayon, David White andTerry Young; sleep-disordered breathing protocol team: Christian Guilleminault, Stuart Quan and David White; EEG/neurocognitive function team: Jed Black, Alan Gevins, Max Hirshkowitz, Gary Kay and Tracy Kuo; mood and sleepiness assessment team: Ruth Benca, William C. Dement, Karl Doghramji, Tracy Kuo and James K. Walsh; quality-of-life assessment team: W. Ward Flemons and Robert M. Kaplan; APPLES secondary analysis-neurocognitive (ASA-NC) team: Dean Beebe, Robert Heaton, Joel Kramer, Ronald Lazar, David Loewenstein and Frederick Schmitt National Heart, Lung, and Blood Institute (NHLBI): Michael J. Twery, Gail G. Weinmann and Colin O. Wu; Data and Safety Monitoring Board (DSMB): seven-year terms: Richard J. Martin (chair), David F. Dinges, Charles F. Emery, Susan M. Harding, John M. Lachin and Phyllis C. Zee; other terms: Xihong Lin (2 years) and Thomas H. Murray (1 year).

\section{References}

1 Strohl KP, Brown DB, Collop N, et al. An official American Thoracic Society Clinical Practice Guideline: sleep apnea, sleepiness, and driving risk in noncommercial drivers. An update of a 1994 Statement. Am J Respir Crit Care Med 2013; 187: 1259-1266.

2 Kapur VK, Baldwin CM, Resnick HE, et al. Sleepiness in patients with moderate to severe sleep-disordered breathing. Sleep 2005; 28: 472-477.

3 Roure N, Gomez S, Mediano O, et al. Daytime sleepiness and polysomnography in obstructive sleep apnea patients. Sleep Med 2008; 9: 727-731.

4 Gottlieb DJ, Whitney CW, Bonekat WH, et al. Relation of sleepiness to respiratory disturbance index: the Sleep Heart Health Study. Am J Respir Crit Care Med 1999; 159: 502-507.

5 Bjorvatn B, Lehmann S, Gulati S, et al. Prevalence of excessive sleepiness is higher whereas insomnia is lower with greater severity of obstructive sleep apnea. Sleep Breath 2015; 19: 1387-1393.

6 Dixon JB, Dixon ME, Anderson ML, et al. Daytime sleepiness in the obese: not as simple as obstructive sleep apnea. Obesity 2007; 15: 2504-2511.

7 Mediano O, Barceló A, de la Peña M, et al. Daytime sleepiness and polysomnographic variables in sleep apnoea patients. Eur Respir J 2007; 30: 110-113.

8 Sharkey KM, Orff HJ, Tosi C, et al. Subjective sleepiness and daytime functioning in bariatric patients with obstructive sleep apnea. Sleep Breath 2013; 17: 267-274.

9 Jacobsen JH, Shi L, Mokhlesi B. Factors associated with excessive daytime sleepiness in patients with severe obstructive sleep apnea. Sleep Breath 2013; 17: 629-635.

10 Pamidi S, Knutson KL, Ghods F, et al. Depressive symptoms and obesity as predictors of sleepiness and quality of life in patients with REM-related obstructive sleep apnea: cross-sectional analysis of a large clinical population. Sleep Med 2011; 12: 827-831.

11 Chen R, Xiong KP, Lian YX, et al. Daytime sleepiness and its determining factors in Chinese obstructive sleep apnea patients. Sleep Breath 2011; 15: 129-135.

12 Budhiraja R, Roth T, Hudgel DW, et al. Prevalence and polysomnographic correlates of insomnia comorbid with medical disorders. Sleep 2011; 34: 859-867.

13 Budhiraja R, Parthasarathy S, Budhiraja P, et al. Insomnia in patients with COPD. Sleep 2012; 35: 369-375.

14 Budhiraja R, Quan SF, Punjabi NM, et al. Power spectral analysis of the sleep electroencephalogram in heartburn patients with or without gastroesophageal reflux disease: a feasibility study. J Clin Gastroenterol 2010; 44: 91-96.

15 Kushida CA, Nichols DA, Quan SF, et al. The Apnea Positive Pressure Long-term Efficacy Study (APPLES): rationale, design, methods, and procedures. J Clin Sleep Med 2006; 2: 288-300.

16 Hamilton M. A rating scale for depression. J Neurol Neurosurg Psychiatr 1960; 23: 56-62.

17 Johns MW. A new method for measuring daytime sleepiness: the Epworth sleepiness scale. Sleep 1991; 14: 540-545. 
18 Johns MW. Sensitivity and specificity of the multiple sleep latency test (MSLT), the maintenance of wakefulness test and the Epworth sleepiness scale: failure of the MSLT as a gold standard. J Sleep Res 2000; 9: 5-11.

19 Doghramji K, Mitler MM, Sangal RB, et al. A normative study of the maintenance of wakefulness test (MWT). Electroencephalogr Clin Neurophysiol 1997; 103: 554-562.

20 Quan SF, Chan CS, Dement WC, et al. The association between obstructive sleep apnea and neurocognitive performance - the Apnea Positive Pressure Long-term Efficacy Study (APPLES). Sleep 2011; 34: 303-314B.

21 Budhiraja R, Kushida CA, Nichols DA, et al. Impact of randomization, clinic visits, and medical and psychiatric cormorbidities on continuous positive airway pressure adherence in obstructive sleep apnea. J Clin Sleep Med 2016; 12: 333-341.

22 Björnsdóttir E, Benediktsdóttir B, Pack AI, et al. The prevalence of depression among untreated obstructive sleep apnea patients using a standardized psychiatric interview. J Clin Sleep Med 2016; 12: 105-112.

23 Bixler EO, Vgontzas AN, Lin HM, et al. Excessive daytime sleepiness in a general population sample: the role of sleep apnea, age, obesity, diabetes, and depression. J Clin Endocrinol Metab 2005; 90: 4510-4515.

24 Grandner MA, Martin JL, Patel NP, et al. Age and sleep disturbances among American men and women: data from the U.S. Behavioral Risk Factor Surveillance System. Sleep 2012; 35: 395-406.

25 Edwards BA, O'Driscoll DM, Ali A, et al. Aging and sleep: physiology and pathophysiology. Semin Respir Crit Care Med 2010; 31: 618-633.

26 Crawford MR, Bartlett DJ, Coughlin SR, et al. The effect of continuous positive airway pressure usage on sleepiness in obstructive sleep apnoea: real effects or expectation of benefit? Thorax 2012; 67: 920-924.

27 Nguyên XL, Rakotonanahary D, Chaskalovic J, et al. Residual subjective daytime sleepiness under CPAP treatment in initially somnolent apnea patients: a pilot study using data mining methods. Sleep Med 2008; 9: 511-516.

28 Pépin JL, Viot-Blanc V, Escourrou P, et al. Prevalence of residual excessive sleepiness in CPAP-treated sleep apnoea patients: the French multicentre study. Eur Respir J 2009; 33: 1062-1067.

29 Veasey SC, Davis CW, Fenik P, et al. Long-term intermittent hypoxia in mice: protracted hypersomnolence with oxidative injury to sleep-wake brain regions. Sleep 2004; 27: 194-201.

30 Fong SY, Ho CK, Wing YK. Comparing MSLT and ESS in the measurement of excessive daytime sleepiness in obstructive sleep apnoea syndrome. J Psychosom Res 2005; 58: 55-60.

31 Budhiraja R, Thomas R, Kim M, et al. The role of big data in the management of sleep-disordered breathing. Sleep Med Clin 2016; 11: 241-255.

32 Budhiraja R, Parthasarathy S, Drake CL, et al. Early CPAP use identifies subsequent adherence to CPAP therapy. Sleep 2007; 30: 320-324. 